\title{
Commentary: Alpha-Synuclein Aggregates Cause Calcium Dysregu- lation by Activating Calcium Pump SERCA
}

\author{
Cristine Betzer ${ }^{1,2}$, Poul Henning Jensen ${ }^{1,2 *}$
}

Danish Research Institute of Translational Neuroscience - DANDRITE, Aarhus University, Denmark

${ }^{2}$ Department of Biomedicine, Aarhus University, Denmark

\section{Article Info}

\section{Article Notes}

Received: June 20, 2018

Accepted: July 06, 2018

\section{*Correspondence:}

Dr. Poul Henning Jensen, Danish Research Institute of

Translational Neuroscience- DANDRITE, Aarhus University, Denmark; Telephone No: +45 28992056;

E-mail: phj@biomed.au.dk.

C 2018 Jensen PH. This article is distributed under the terms of the Creative Commons Attribution 4.0 International License
We have recently reported that alpha-synuclein aggregates induce calcium dysregulation by activating the calcium pump, SERCA in the endoplasmic reticulum ${ }^{1}$. The dysregulation presents as a biphasic change in cytosolic calcium with an initial phase with $20 \%$ decreased calcium followed by final phase with increased calcium. These findings are novel with respect to; 1) demonstrating activation of SERCA as a gain-offunction acquired by aggregated alpha-synuclein; 2) demonstrating an initial phase with reduced cytosolic calcium in neurons that experience a build-up of alpha-synuclein aggregates. The initial phase occurs when cells appear morphologically unaffected and precedes the wellknown later phase with increased cytosolic calcium and subsequent cell death. Although the reduction in cytosolic calcium of $20 \%$ may appear modest are its short and long-term consequences largely unknown because this phase not previously has been described. However, both the early and late phase of the calcium dysregulation can be antagonized pharmacologically by inhibiting SERCA and this treatment restored the survival of the cells to the level of the control cells not being subject to alpha-synuclein aggregate stress. The interaction between aggregated synuclein and SERCA could be demonstrated in neurons in brain tissue affected by dementia with Lewy bodies supporting that the initial phase with reduced cytosolic calcium is relevant for human pathology. We hypothesize the alpha-synuclein aggregate induced stimulation of SERCA and the early-phase with reduced cytosolic calcium will compromise neurons ability to support their role in normal circuitries, integrated brain functions and ultimately cause symptoms. This phase may exist for prolonged periods before the neurons enters the latephase with increased cytosolic calcium that has been studied according to the original "calcium hypothesis",2,3. Understanding the mechanisms whereby the abnormal activation of SERCA and its down-stream dysregulation of calcium contributes to neuronal dysfunction and eventual cell death holds potential for new symptomatic and disease modifying strategies for synucleinopathies.

\section{Calcium Disturbances and Parkinson's Disease}

The so-called "calcium hypothesis" has for 3 decades been used as a basis to understand the vulnerability of the aging brain by mechanisms that also may apply to late onset neurodegenerative diseases ${ }^{2,3}$. Central to this hypothesis is that abnormal increases in cytosolic calcium plays a pivotal and detrimental role. The calcium hypothesis has received special attention in Parkinson's disease where the motor symptoms are associated to loss of the dopaminergic neurons in substantia nigra pars compacta. These dopaminergic neurons have a large terminal field and 
display autonomous pace-making firing, which lead to a large influx of calcium ions via L-type voltage gated calcium channels (CaV). Maintaining the low normal cytosolic calcium level requires active transport processes and a large mitochondrial ATP production, which carry an inherent risk of oxidative stress ${ }^{4-6}$. The risk of developing Parkinson's disease is reduced in patients treated for hypertension with L-type calcium channel blockers targeting the $\mathrm{CaV}$ channels $^{7-9}$. However, the treatment did not reduce the risk of developing dementia and the length of previous use of the calcium channel blockers prior to diagnosis was not associated with reduced risk of $\mathrm{PD}^{9}$. This led the authors suggest the treatment was associated with symptomatic relief rather than preventing disease development ${ }^{9}$ and may represent a modulations of the late phase of our hypothesis with increased calcium. The expression of L-type calcium channels is changed in brains affected by Parkinson's disease and the changes could be observed in early stage patients and in areas not expected to carry alpha-synuclein aggregate pathology ${ }^{10}$. These changes in $\mathrm{CaV}$ channel expression can hypothetically represent compensatory responses to alpha-synuclein aggregates stimulating SERCA and decreasing cytosolic calcium.

\section{Calcium Dysregulation - Too High or Too Low?}

The calcium hypothesis describes calcium dysregulation as increased cytosolic calcium, but our finding illustrates that cytosolic calcium can also be too low in order for neurons to maintain their functionality. There are other reports suggesting that elevation of cytosolic calcium actually protectsperipheral neurons and spinalganglion ${ }^{11-13}$. Furthermore, stimulation of synaptic NMDA stimulated calcium channels induces an increase in nuclear transients with increased calcium levels that activates transcription of so-called neuroprotective "neuronal shield" genes ${ }^{14-20}$. A reduction in the cytosolic calcium will likely reduce the efficacy of this protective calcium-dependent synapseto-nucleus mechanism because the fenestrated nuclear membrane will allow diffusion of calcium into the cytosol thereby yielding a reduced nuclear calcium level. Moreover, stimulation of SERCA by aggregated alpha-synuclein overloads the endoplasmic reticulum with calcium, which can trigger a host of fundamental responses, including unfolded protein response, mitochondrial calcium filling, store-operated calcium entry ${ }^{21}$, dysregulation of presynaptic neurotransmitter release $\mathrm{e}^{22}$ and activity of specialized overload calcium channels such as TMCO123.

SERCA Activation, Presenilins and Calcium in the Endoplasmic Reticulum: Similarities between Synucleinopathies and Alzheimer's Disease

Some familial forms of Alzheimer's disease are linked to mutations in presenilins. Presenilins 1 and 2 are transmembrane proteins in the endoplasmic reticulum that regulate the production of amyloidogenic $A \beta$ peptides ${ }^{24}$. Presenilins functions as low conductance calcium leak channels, that reduce the calcium load in the endoplasmic reticulum ${ }^{25}$ and this effect is balanced by their function as physiological activators of SERCA, which pumps calcium back into the endoplasmic reticulum ${ }^{30}$. This balance is destroyed in cells from families carrying Alzheimer's disease-causing mutations in their presenilins that block their calcium leak channel properties, which will tend to lower their neuronal cytosolic calcium. Such Alzheimer's disease patients may share pathophysiogical signaling mechanisms with Parkinson's disease patients that also exhibit a phase with reduced cytosolic calcium in affected neurons and may comprise dysfunctions in store-operated calcium entry, expression of ryanodine receptors, and inositol trisphosphate receptors ${ }^{26-29}$.

Further studies into our "Calcium hypothesis, version 2" centered around mechanisms initiated by reduced cytosolic calcium and calcium overload in the endoplasmic reticulum hold potential for generating novel therapeutic strategies for both symptomatic relief and true disease modification in Parkinson's disease and subgroups of Alzheimer's disease.

\section{Acknowledgements}

The work is supported by the Lundbeck Foundation (Grant no. R223-2015-4222, and R248-2016-2518), Danish Research Institute of Translational Neuroscience DANDRITE, and Aarhus University.

\section{References}

1. Betzer C, Lassen LB, Olsen A, et al., Alpha-synuclein aggregates activate calcium pump SERCA leading to calcium dysregulation. EMBO Rep. 2018; 19(5).

2. Khachaturian ZS. Towards theories of brain aging, in Handbook of Studies on Psychiatry and Old Age, D.S.B. Kay, G.W, Editor. Elsevier. 1984.

3. Berridge MJ. Calcium hypothesis of Alzheimer's disease. Pflugers Arch. 2010; 459(3): p. 441-9.

4. Surmeier DJ. Calcium ageing and neuronal vulnerability in Parkinson's disease. Lancet Neurol. 2007; 6(10): p. 933-8.

5. Dryanovski DI, Guzman JN, Xie Z, et al. Calcium entry and alpha-synuclein inclusions elevate dendritic mitochondrial oxidant stress in dopaminergic neurons. J Neurosci. 2013; 33(24): p. 10154-64.

6. Surmeier DJ, Obeso JA, Halliday GM. Selective neuronal vulnerability in Parkinson disease. Nat Rev Neurosci. 2017; 18(2): p. 101-113.

7. Becker C, Jick SS, Meier CR. Use of antihypertensives and the risk of Parkinson disease. Neurology. 2008; 70(16 Pt 2): p. 1438-44.

8. Ritz B, Rhodes SL, Qian L, et al. L-type calcium channel blockers and Parkinson disease in Denmark. Ann Neurol. 2010; 67(5): p. 600-6. 
9. Pasternak B, Svanström H, Nielsen NM, et al. Use of calcium channel blockers and Parkinson's disease. Am J Epidemiol. 2012; 175(7): p. 627-35.

10. Hurley MJ, Brandon B, Gentleman SM, et al. Parkinson's disease is associated with altered expression of CaV1 channels and calcium-binding proteins. Brain. 2013; 136(Pt 7): p. 2077-97.

11. Scott BS, Fisher KC. Potassium concentration and number of neurons in cultures of dissociated ganglia. Exp Neurol. 1970; 27(1): p. 16-22.

12. Franklin JL, Sanz-Rodriguez C, Juhasz A, et al. Chronic depolarization prevents programmed death of sympathetic neurons in vitro but does not support growth: requirement for Ca2+ influx but not Trk activation. J Neurosci. 1995; 15(1 Pt 2): p. 643-64.

13. Miller AL, Prieskorn DM, Altschuler RA, et al. Mechanism of electrical stimulation-induced neuroprotection: effects of verapamil on protection of primary auditory afferents. Brain Res. 2003; 966(2): p. 218-30.

14. Mao Z, Bonni A, Xia F, et al. Neuronal activity-dependent cell survival mediated by transcription factor MEF2. Science. 1999; 286(5440): p. 785-90.

15. Hardingham GE, Fukunaga Y, Bading H. Extrasynaptic NMDARs oppose synaptic NMDARs by triggering CREB shut-off and cell death pathways. Nat Neurosci. 2002; 5(5): p. 405-14.

16. Papadia S, Stevenson P, Hardingham NR, et al. Nuclear Ca2+ and the $C A M P$ response element-binding protein family mediate a late phase of activity-dependent neuroprotection. J Neurosci. 2005; 25(17): p. 4279-87.

17. Lee B, Butcher GQ, Hoyt KR, et al. Activity-dependent neuroprotection and $c A M P$ response element-binding protein (CREB): kinase coupling, stimulus intensity, and temporal regulation of CREB phosphorylation at serine 133. J Neurosci. 2005; 25(5): p. 1137-48.

18. Bok J, Wang Q Huang J, et al. CaMKII and CaMKIV mediate distinct prosurvival signaling pathways in response to depolarization in neurons. Mol Cell Neurosci. 2007; 36(1): p. 13-26.
19. Zhang SJ, Steijaert MN, Lau D, et al. Decoding NMDA receptor signaling: identification of genomic programs specifying neuronal survival and death. Neuron. 2007; 53(4): p. 549-62.

20. Bading H. Nuclear calcium signalling in the regulation of brain function. Nat Rev Neurosci. 2013; 14(9): p. 593-608.

21. Carreras-Sureda A, Pihán P, Hetz C. Calcium signaling at the endoplasmic reticulum: fine-tuning stress responses. Cell Calcium. 2018; 70: p. 24-31.

22. de Juan-Sanz J, Holt GT, Schreiter ER, et al. Axonal Endoplasmic Reticulum $\mathrm{Ca}(2+)$ Content Controls Release Probability in CNS Nerve Terminals. Neuron. 2017; 93(4): p. 867-881 e6.

23. Wang QC, Zheng $\mathrm{Q}$ Tan $\mathrm{H}$, et al. TMCO1 Is an ER Ca(2+) LoadActivated Ca(2+) Channel. Cell. 2016; 165(6): p. 1454-1466.

24. Tolia A, De Strooper B. Structure and function of gammasecretase. Semin Cell Dev Biol. 2009; 20(2): p. 211-8.

25. Tu H, Nelson 0, Bezprozvanny A, et al. Presenilins form ER Ca2+ leak channels, a function disrupted by familial Alzheimer's disease-linked mutations. Cell. 2006; 126(5): p. 981-93.

26. Blalock EM, Chen KC, Sharrow K, et al. Gene microarrays in hippocampal aging: statistical profiling identifies novel processes correlated with cognitive impairment. J Neurosci. 2003; 23(9): p. 3807-19.

27. Emilsson L, Saetre P, Jazin E. Alzheimer's disease: $m R N A$ expression profiles of multiple patients show alterations of genes involved with calcium signaling. Neurobiol Dis. 2006; 21(3): p. 618-25.

28. Bezprozvanny I. Calcium signaling and neurodegenerative diseases. Trends Mol Med. 2009; 15(3): p. 89-100.

29. Popugaeva E, Bezprozvanny I. Can the calcium hypothesis explain synaptic loss in Alzheimer's disease. Neurodegener Dis. 2014; 13(2-3): p. 139-41.

30. Green KN, Demuro A, Akbari Y, et al. SERCA pump activity is physiologically regulated by presenilin and regulates amyloid beta production. J Gen Physiol. 2008; 132(2): p. i1. 